\title{
FABRICATION AND EVALUATION OF SOLID DISPERSION CONTAINING GLIBENCLAMIDE
}

\author{
NIKITA SEHGAL, VISHAL GUPTA N, GOWDA DV*, PRAVEEN SIVADASU
}

Department of Pharmaceutics, JSS College of Pharmacy, Sri Shivarathreeshwara Nagara, Mysuru, JSS Academy of Higher Education and Research, JSS Medical Institutions Campus, Sri Shivarathreeshwara Nagara, Mysuru - 570 015, Karnataka, India. Email: dvgowda@jssuni.edu.in

Received: 24 April 2018, Revised and Accepted: 24 April 2018

ABSTRACT

Objective: The aim of the present study was to increase the dissolution rate of glibenclamide (GLIB) by molecular dispersion of drug in the polymeric matrix of Pluronic F-127.

Methods: GLIB-loaded solid dispersions were formulated by fusion method. The formulated solid dispersions were characterized for scanning electron microscopy (SEM), X-ray diffractometry (XRD), differential scanning calorimetry (DSC), and evaluated for percentage yield, drug content, solubility, and in vitro dissolution profile, and stability studies were conducted as per International Conference on Harmonisation guidelines Q1A in stability chamber, both at intermediate and accelerated conditions.

Results: Both XRD and DSC studies suggested that crystalline GLIB was converted to amorphous form after loading into carrier. SEM studies revealed that the prepared solid dispersions were in the form of irregular particles with the absence of crystalline material. Due to this conversion of crystalline to amorphous state, formulated solid dispersions had shown improved dissolution rate profile of GLIB and stability studies suggested that formulated solid dispersions showed no significant changes in appearance and also in drug content.

Conclusion: Thus, from the obtained results, it can be concluded that dissolution profile of GLIB can be improved by formulating as solid dispersion.

Keywords: Glibenclamide, Pluronic F-127, Solid dispersion, Diabetes.

(C) 2018 The Authors. Published by Innovare Academic Sciences Pvt Ltd. This is an open access article under the CC BY license (http://creativecommons. org/licenses/by/4. 0/) DOI: http://dx.doi.org/10.22159/ajpcr.2018.v11i8.26236

\section{INTRODUCTION}

Solid dispersions are prepared to enhance the dissolution profile of drugs by dispersing drug in an inert matrix. Matrix can either be amorphous or crystalline in which drug will be distributed molecularly, as crystalline or amorphous particles [1]. Dissolution and solubility are two important parameters which alter oral bioavailability of any drug, thus efforts should be done to improve the solubility and dissolution rate is essential. Characteristics of the drugs can be altered by converting them into salt forms, size reduction, and by adding surfactants. In the current research, Pluronic F-127 was selected as carrier and solid dispersions were developed by employing solvent evaporation method $[2,3]$.

Among various parameters which have a direct impact on bioavailability solubility and permeability were considered as most crucial parameters, other parameters include rate of dissolution, first pass metabolism, and liability for efflux mechanisms [4]. In recent times, different techniques were used to improve the bioavailability of the drug such as reduction of particle size, physical modifications, changing the crystalline nature of the drug, usage of cyclodextrin complexes, and solid dispersions. Among these various techniques, solid dispersions grabbed the attention of scientists as it possesses certain advantages such as enhancing dissolution rate, absorption, and reduction of pre-metabolism when compared to other techniques. In general, solid dispersion consists of two parts one is hydrophilic matrix and a lipophilic drug [5-8].

Glibenclamide (GLIB) is a poorly soluble drug that belongs to the class of sulfonylurea, which is prescribed to treat type-II diabetes where hyperglycemia cannot be adequately managed with exercise and diet and usually recommended for the patients who have not responded to the maximal dose of metformin. It is an antidiabetic drug in a class of medications known as sulfonylureas. It is poorly soluble in gastrointestinal fluid [9]. GLIB has poor solubility with formulationdependent bioavailability. Therefore, in this research, solid dispersion was prepared by fusion method to improve bioavailability of GLIB [10].

\section{MATERIALS AND METHODS}

Materials

GLIB was obtained from Yarrow Chem Product - Mumbai. Pluronic F-127 was obtained from Sigma-Aldrich, India. Sodium hydroxide and potassium dihydrogen phosphate were purchased from SD Fine Chemicals Limited, Mumbai.

\section{Methods}

Preparation of GLIB solid dispersion

GLIB-loaded solid dispersions were done by fusion method. Solid dispersion with carrier, i.e., Pluronic F-127 was made in different formulation F1-F8 (as shown in Table 1). Pluronic F-127 was melted at $70 \pm 4^{\circ} \mathrm{C}$ for $7-8 \mathrm{~min}$ in porcelain dish on water bath and with constant stirring to the molten carrier, powdered drug was added. The acquired solid dispersion was solidified at room temperature and was kept in desiccators for $24 \mathrm{~h}$. After $24 \mathrm{~h}$ of storage, solid dispersion was passed through sieved no. 100 [11].

\section{Characterization of GLIB solid dispersion}

$X$-ray diffractometry $(X R D)$

Crystallinity of GLIB was determined using XRD analysis (Bruker D8 $\mathrm{XRD)}$ and its respective solid dispersions.

\section{Differential scanning calorimetry (DSC)}

All DSC studies of pure drug and its solid dispersion formulations were done on Shimadzu DSC-TSW 60 thermal analyzer. A few mg of sample 
was entirely sealed into aluminum pans and under nitrogen atmosphere was heated at constant temperature of $10^{\circ} \mathrm{C} / \mathrm{min}$ [12].

\section{Scanning electron microscope (SEM) studies}

Using Hitachi Noran System 7 manufactured by Thermo Fisher Scientific, surface morphology of GLIB solid dispersion was obtained at the appropriate magnification and room temperature.

\section{Percent yield}

The percent yield of GLIB solid dispersions was determined using the following formula [13]:

$$
\% \text { yield }=\frac{\text { Weight of prepared solid dispersion }}{\text { Weight of drug }+ \text { carrier }} * 100
$$

\section{Drug content}

Formulated solid dispersion equivalent to $10 \mathrm{mg}$ was taken and dissolved in $10 \mathrm{ml}$ of methanol. The obtained solution was diluted appropriately in methanol and was examined by UV-visible spectrophotometer (Shimadzu UV-1700) at $229 \mathrm{~nm}$ [13].

\section{Saturation solubility studies}

In a glass-stoppered flask containing $10 \mathrm{ml}$ of $\mathrm{pH} 1.2$ hydrochloric acid ( $\mathrm{HCl}$ ) buffer with $0.1 \%$ sodium lauryl sulfate (SLS), surplus amount of the drug and its solid dispersion were added and additionally $7.2 \mathrm{pH}$ phosphate buffer was also added. Using UV-visible spectrophotometer (Shimadzu UV-1700), solubility of drug was calculated at $229 \mathrm{~nm} \mathrm{[2].}$

\section{Dissolution study of GLIB solid dispersions}

Weight of solid dispersion equivalent to $10 \mathrm{mg}$ of GLIB was taken and using $900 \mathrm{ml}$ of $\mathrm{pH} 7.2$ phosphate buffer as dissolution medium and dissolution study was done in six stages employing USP Apparatus II (rotating paddle type - Electrolab TDT-08L, India) at stirring speed of $75 \mathrm{rpm}$ and at controlled temperature of $37 \pm 0.5^{\circ} \mathrm{C} .5 \mathrm{ml}$ sample was withdrawn at predetermined interval and same volume of distilled water was added to it. Using UV spectrophotometer apparatus (Shimadzu UV-1700) at $229 \mathrm{~nm}$, all the samples were analyzed against appropriate blank [14].

\section{Stability study}

The stability study of the formulations was done according to International Conference on Harmonisation guideline Q1AR2 in stability chamber (Thermo Lab, Mumbai). The stability study was performed at intermediate and accelerated conditions in closed containers at specific storage conditions (Table 2). In both cases, samples were analyzed for drug content at 0,3 , and 6 months to find out the effect of temperature and humidity on product stability.

\section{RESULTS AND DISCUSSION}

\section{Characterization of GLIB solid dispersions}

XRD studies

XRD of GLIB API, pure Pluronic F-127 polymer, and solid dispersion formulation F7 is shown in Fig. 1. The peaks of GLIB API were found at $2 \Theta$ angles of $12.95,16.46,19.38,21.9$, and $27.55^{\circ}$, indicating its crystalline nature. In contrast, the solid dispersion formulation F7 showed reduction in intensity of major peaks and disappearance of minor peaks, indicating the conversion of crystalline drug into semicrystalline drug into semi-crystalline nature. As crystallinity of drug decreases, it may contribute to the enhancement of dissolution of drug [2-4].

\section{DSC studies}

DSC thermogram of GLIB API, pure Pluronic F-127, and optimized formulation of solid dispersion is shown in Fig. 2. The DSC curve of pure GLIB API and pure Pluronic F-127 showed sharp endothermic peaks at $140.12^{\circ} \mathrm{C}$ and $54.57^{\circ} \mathrm{C}$, respectively. Within the DSC thermogram of solid dispersion, the endothermic peak corresponding to the melting of GLIB was absent. This might be due to the dissolution of crystalline GLIB into the molten carrier or translation of GLIB to amorphous form from crystalline [9].

\section{SEM studies}

The SEM images for images for GLIB API and solid dispersion formulation F7 are shown in Fig. 3a and b. The GLIB API microphotograph (Fig. 3a) shows crystalline rectangular shape nature, whereas an image of solid dispersion (Fig. 3b) reveals irregular particles with the absence of any crystalline material.

\section{Evaluation of GLIB solid dispersions \\ Percent yield and drug content}

Percentage yield of solid dispersions was calculated and reported in Table 3. The highest percentage yield $(98.41 \pm 0.87 \%)$ was found in solid dispersion F6 and lowest yield was (88.19 1 1.43) observed in F2. As seen in Table 3, the drug content analysis showed that the drug loading was high (>87\%), and there was proper distribution of the drug in the carrier, i.e., Pluronic F-127. The drug content evaluation of different batches of solid dispersion showed that the drug content was in the range of $89.24 \pm 0.94-98.41 \pm 0.87 \%$ of the total amount of the drug added to carrier.

\section{Saturation solubility studies}

The saturation solubility of the GLIB solid dispersion containing drug and carrier in different ratios, in pH $1.2 \mathrm{HCL}$ buffer with $0.1 \%$ SLS and phosphate buffer of pH 7.2 is graphically represented in Fig. 4. The solubility values of solid dispersion for formulations were found to be in the range of $29.21-108.61 \mu \mathrm{g} / \mathrm{ml}$ and $34.13-117.9 \mu \mathrm{g} / \mathrm{ml}$, respectively, in pH 1.2 HCL buffer with $0.1 \%$ SLS and pH phosphate buffer of 7.2. Due to Pluronic F-127, there was formation of complex between watersoluble polymeric carrier and poorly soluble drug and second might be due to increased wettability of GLIB, there was an increase in solubility of GLIB. Among all the prepared of solid dispersions, on the basis of solubility, it was found that formulation F7 has adequate solubility. Based on the solubility data obtained, the solid dispersion F7 can be used for the further studies.

\section{Dissolution study of GLIB solid dispersions}

Results obtained from dissolution studies suggested that solid dispersions loaded with GLIB showed an improved dissolution in $\mathrm{pH} 7.2$ phosphate buffer. As shown in Fig. 5, it was evident that formulation F7 had shown improved dissolution rate when compared with other formulations. From the results, it can be inferred that dissolution rate of

Table 1: Formulation of Glibenclamide solid dispersion

\begin{tabular}{ll}
\hline Formulation code & Drug: Carrier ratio \\
\hline F1 & $1: 0.5$ \\
F2 & $1: 1$ \\
F3 & $1: 1.5$ \\
F4 & $1: 2$ \\
F5 & $1: 2.5$ \\
F6 & $1: 3$ \\
F7 & $1: 3.5$ \\
F8 & $1: 4$ \\
\hline
\end{tabular}

Table 2: Different stability study condition according to the ICH guideline

\begin{tabular}{llll}
\hline Study & Storage condition & Minimum time period & Sampling interval \\
\hline Intermediate $(\mathrm{IM})$ & $30 \pm 2^{\circ} \mathrm{C} / 65 \% \mathrm{RH} \pm 5 \% \mathrm{RH}$ & 6 months & 0,3 , and 6 months \\
Accelerated $(\mathrm{AC})$ & $40 \pm 2^{\circ} \mathrm{C} / 75 \% \mathrm{RH} \pm 5 \% \mathrm{RH}$ & 6 months & 0,3 , and 6 months \\
\hline
\end{tabular}

ICH: International Conference on Harmonisation 


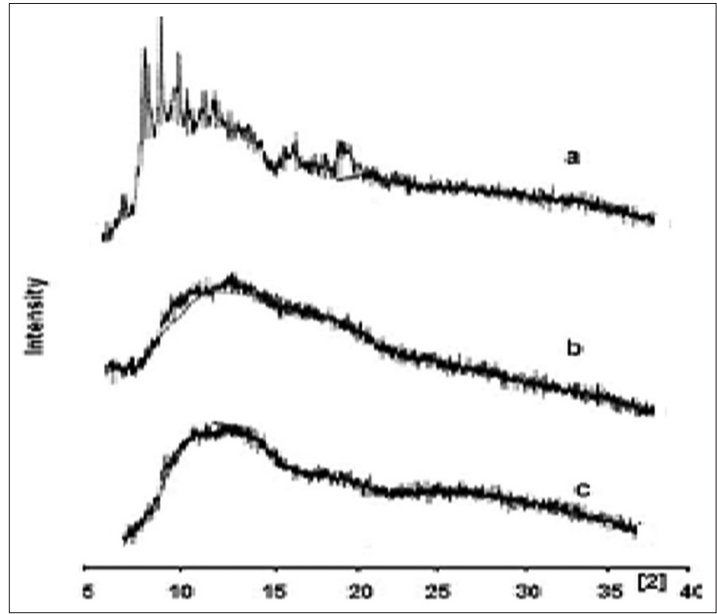

Fig. 1: X-ray diffractometry studies of (a) pure drug and formulated solid dispersions (b) F1 (c) F7

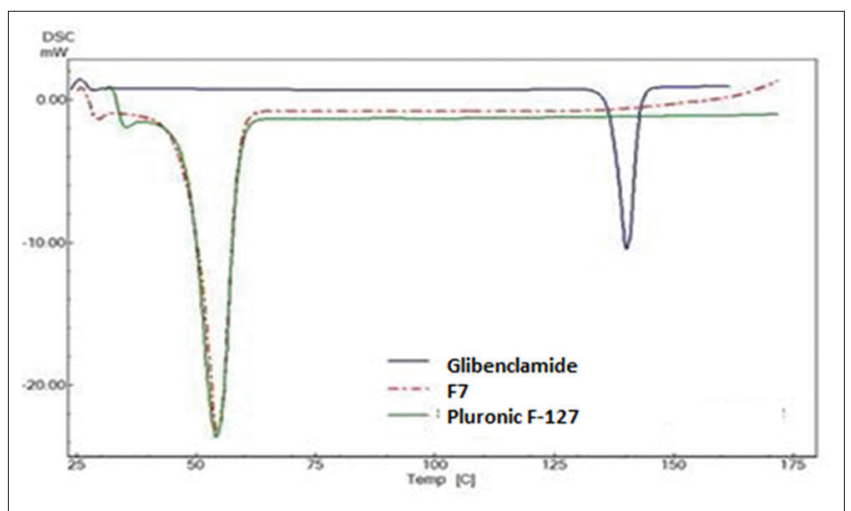

Fig. 2: Differential scanning calorimetry thermogram of glibenclamide, pure Pluronic F-127 carrier, and optimized solid dispersion formulation
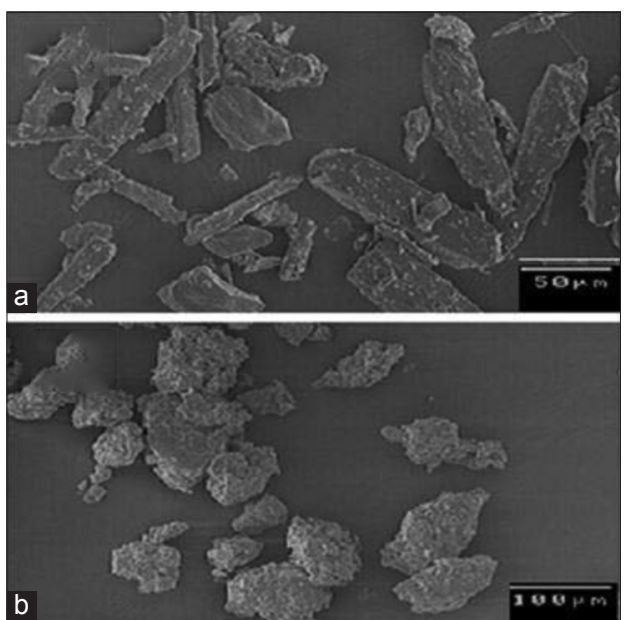

Fig. 3: ( $a$ and b) Scanning electron microscopy images for pure drug and solid dispersion

GLIB increased with increase of carrier concentration due to increased wettability of drug in the carrier.

\section{Stability studies}

The GLIB formulation was subjected for stability studies; formulations were kept at intermediate and accelerated condition in stability

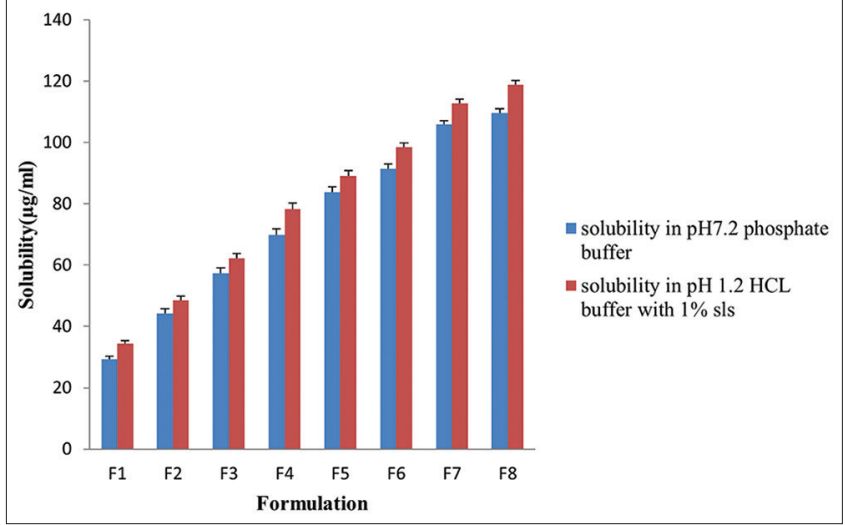

Fig. 4: Solubility studies of glibenclamide solid dispersion formulations in $\mathrm{pH} 1.2 \mathrm{HCl}$ buffer and $\mathrm{pH} 7.2$ phosphate buffer

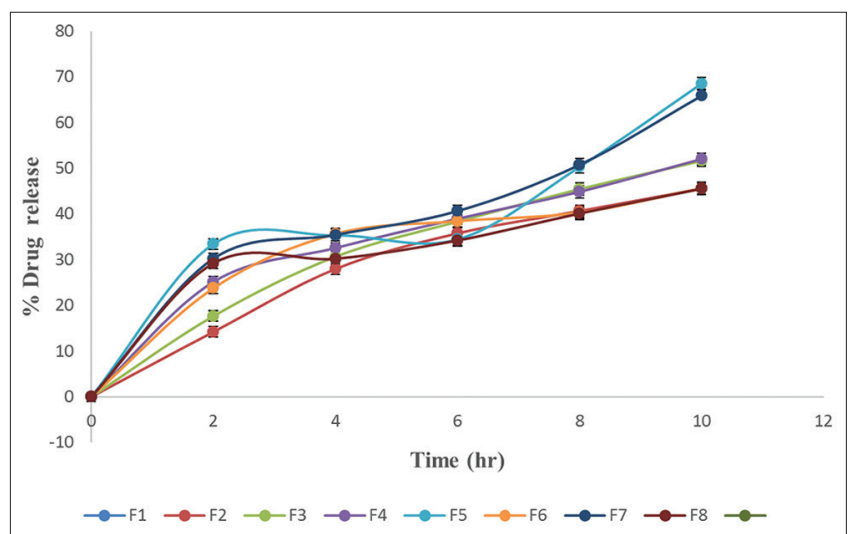

Fig. 5: Dissolution studies of glibenclamide solid dispersion

Table 3: Percentage yield of solid dispersions

\begin{tabular}{lll}
\hline Formulation code & Percentage yield (\%) & Drug content (\%) \\
\hline F1 & $86.12 \pm 1.22$ & $88.93 \pm 1.87$ \\
F2 & $89.24 \pm 0.94$ & $90.24 \pm 1.06$ \\
F3 & $94.51 \pm 1.59$ & $89.11 \pm 1.54$ \\
F4 & $89.68 \pm 1.02$ & $92.13 \pm 1.23$ \\
F5 & $96.78 \pm 1.10$ & $91.86 \pm 1.73$ \\
F6 & $98.41 \pm 0.87$ & $92.89 \pm 0.95$ \\
F7 & $91.05 \pm 1.06$ & $87.13 \pm 1.49$ \\
F8 & $88.19 \pm 1.43$ & $90.83 \pm 1.15$ \\
\hline
\end{tabular}

Mean \pm SD, n=3. SD: Standard deviation

chamber. Samples were taken out on interval of 0, 3, and 6 months and test for the percentage drug content was conducted. The results showed no significant changes in appearance and also in drug content as depicted in Table 4. Highest degradation was observed in formulation F5 after 6 months in accelerated condition. Hence, it can be said that the formulations are stable at various storage conditions for longer periods.

\section{CONCLUSION}

Fusion technique was used to prepare solid dispersion of GLIB with Pluronic F-127. This technique helped to increase dissolution of GLIB. The stability of GLIB was not affected by dispersing it with Pluronic F-127 carrier. Formulation can be stored for long time without degradation. The preparation F7 showed optimum dissolution. Thus, it can be concluded that GLIB solid dispersion can be useful in increasing the dissolution and improvement of bioavailability of the drug. 
Table 4: Stability profile of glibenclamide solid dispersions

\begin{tabular}{|c|c|c|c|c|c|c|}
\hline \multicolumn{7}{|c|}{ (\%) Drug content } \\
\hline \multirow[t]{2}{*}{ Formulation } & \multicolumn{2}{|l|}{0 month } & \multicolumn{2}{|l|}{3 months } & \multicolumn{2}{|l|}{6 months } \\
\hline & IM & AC & IM & AC & IM & AC \\
\hline F1 & $97.2 \pm 1.25$ & $97.2 \pm 1.19$ & $98.1 \pm 1.26$ & $98.3 \pm 0.99$ & $98.1 \pm 0.78$ & $97.9 \pm 1.18$ \\
\hline $\mathrm{F} 2$ & $97.8 \pm 1.02$ & $97.4 \pm 0.96$ & $98.5 \pm 0.98$ & $99.2 \pm 0.98$ & $97.8 \pm 1.23$ & $98.1 \pm 1.79$ \\
\hline F3 & $98.2 \pm 0.95$ & $98.1 \pm 1.23$ & $98.8 \pm 1.23$ & $98.4 \pm 1.32$ & $99.7 \pm 0.98$ & $98.5 \pm 1.39$ \\
\hline $\mathrm{F} 4$ & $99.4 \pm 1.2$ & $99.2 \pm 0.99$ & $99.5 \pm 1.34$ & $97.4 \pm 1.54$ & $98.6 \pm 1.35$ & $98.4 \pm 1.37$ \\
\hline F5 & $97.4 \pm 1.15$ & $97.7 \pm 1.35$ & $98.3 \pm 1.26$ & $98.4 \pm 1.45$ & $99.2 \pm 1.27$ & $97.1 \pm 1.29$ \\
\hline F6 & $99.4 \pm 1.24$ & $99.3 \pm 1.34$ & $97.5 \pm 0.99$ & $98.5 \pm 1.25$ & $97.5 \pm 1.29$ & $98.3 \pm 1.25$ \\
\hline F7 & $98.6 \pm 0.99$ & $98.4 \pm 1.25$ & $96.5 \pm 1.24$ & $98.4 \pm 1.56$ & $99.3 \pm 1.45$ & $97.3 \pm 1.29$ \\
\hline F8 & $99.0 \pm 1.14$ & $98.5 \pm 1.32$ & $98.3 \pm 1.35$ & $96.6 \pm 1.23$ & $98.6 \pm 1.36$ & $98.1 \pm 1.38$ \\
\hline
\end{tabular}

Mean \pm SD, $n=3$. SD: Standard deviation

\section{ACKNOWLEDGMENTS}

The authors express their gratitude to the JSS Academy of Higher Education and Research and JSS College of Pharmacy, Mysuru, for providing necessary support in due course of the work.

\section{AUTHOR'S CONTRIBUTIONS}

Author is a faculty in division of pharmaceutics and the work contributed on faculty development program in the institution.

\section{CONFLICTS OF INTEREST}

The author confirms that this article content has no conflicts of interest.

\section{REFERENCES}

1. Ford JL. The current status of solid dispersions. Pharm Acta Helv 1986;61:69-88.

2. Akiladevi D, Shanmugapandiyan P, Jebasingh D, Basak S. Preparation and evaluation of paracetamol by solid dispersion technique. Int $\mathbf{J}$ Pharm Pharm Sci 2011;3:188-91

3. Sharma DK. Solubility enhancement strategies for poorly water-soluble drugs in solid dispersions: A review. Asian J Pharm 2016;1:9-19.

4. Savjani KT, Gajjar AK, Savjani JK. Drug solubility: Importance and enhancement techniques. ISRN Pharm 2012;2012:1-10.

5. Serajuddin A. Solid dispersion of poorly water-soluble drugs: Early promises, subsequent problems, and recent breakthroughs. J Pharm Sci 1999;88:1058-66.

6. Dhirendra K, Lewis S, Udupa N, Atin K. Solid dispersions: A Review. Pak J Pharm Sci 2009;22:234-46.

7. Allawadi D, Singh N, Singh S, Arora S. Solid dispersions: A review on drug delivery system and solubility enhancement. Int J Pharm Sci Res 2013:4:2094-105.

8. Kurmi R, Mishra DK, Jain DK. Solid dispersion: A novel means of solubility enhancement. J Crit Rev 2016:3:1-8.

9. Alkarib SY, Nur AO. Gastroretentive controlled release glibenclamide oral tablet formulation. Am J Adv Drug Deliv 2017;5:19-37.

10. Sheppard DN, Robinson KA. Mechanism of glibenclamide inhibition of cystic fibrosis transmembrane conductance regulator Cl-channels expressed in a murine cell line. J Physiol 1997;503:333-46.

11. Yadav S, Veena M, Srinivas M. Solid dispersion technique to enhance the solubility and dissolution rate of Aripiprazole by fusion method. Int J Pharm Pharm Sci 2016;8:187-92.

12. Preethi GB, Banerjee S, Shivakumar HN, Kumar MR. Formulation of fast-dissolving tablets of Doxazosin mesylate drug by direct compression method. Int J Appl Pharm 2017;9:22-8.

13. Kar A, Ahmed AB. Enhancement of solubility and dissolution of ibuprofen by solid dispersion technique and formulation of sustained release tablets containing the optimised batch of solid dispersion. Int J Curr Pharm Res 2017;9:37-44.

14. Kaushik S, Pathak K. Development and evaluation of monolithic osmotic tablet of Ketoprofen: Using solid dispersion technique. Int J Pharm Pharm Sci 2016;8:41-7. 